\title{
Pentadecanuclear Fe-containing Polyoxometalate Catalyst for Visible-Light-Driven Generation of Hydrogen
}

Tingting Cui,${ }^{\dagger}$ Lin Qin,${ }^{\dagger}$ Fangyu Fu,${ }^{\dagger}$ Xing Xin,${ }^{\dagger}$ Huijie Li,${ }^{\dagger}$ Xikui Fang $₫$ and Hongjin $\mathrm{Lv}^{* \dagger}$

${ }^{\dagger}$ MOE Key Laboratory of Cluster Science, School of Chemistry and Chemical Engineering, Beijing Institute of Technology, Beijing 102488, P. R. China.

$\$$ School of Chemistry and Chemical Engineering, Harbin Institute of Technology, Harbin 150001, P. R. China.

Corresponding author Email: hlv@bit.edu.cn 


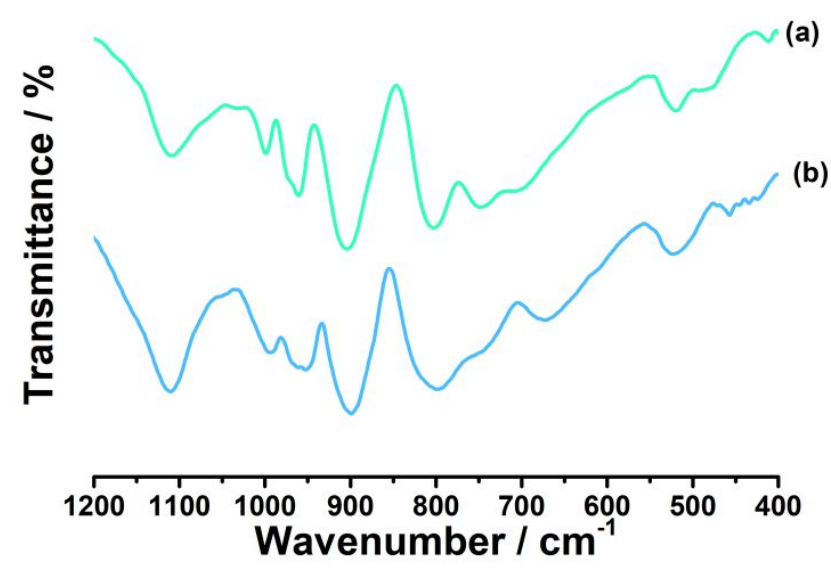

Figure S1. FT-IR spectra of (a) $\mathrm{Na}_{21}-\mathbf{F e}_{15} \mathbf{P}_{\mathbf{4}}\left(\mathbf{S i W}_{\mathbf{9}}\right)_{\mathbf{4}}$ (green) and (b) TBA- $\mathbf{F e}_{15} \mathbf{P}_{\mathbf{4}}\left(\mathbf{S i W}_{\mathbf{9}}\right)_{\mathbf{4}}$ (blue), $\sim 2 \mathrm{wt} \%$ in $\mathrm{KBr}$. The spectra show all the characteristic bands of polyanion $\mathbf{F e}_{15} \mathbf{P}_{\mathbf{4}}\left(\mathbf{S i W}_{\mathbf{9}}\right)_{4}$ confirming that the cation exchange $\left(\mathrm{TBA}^{+}\right.$for $\left.\mathrm{Na}^{+}\right)$does not change the structure of $\mathbf{F e}_{15} \mathbf{P}_{\mathbf{4}}\left(\mathrm{SiW}_{\mathbf{9}}\right)_{\mathbf{4}}$.

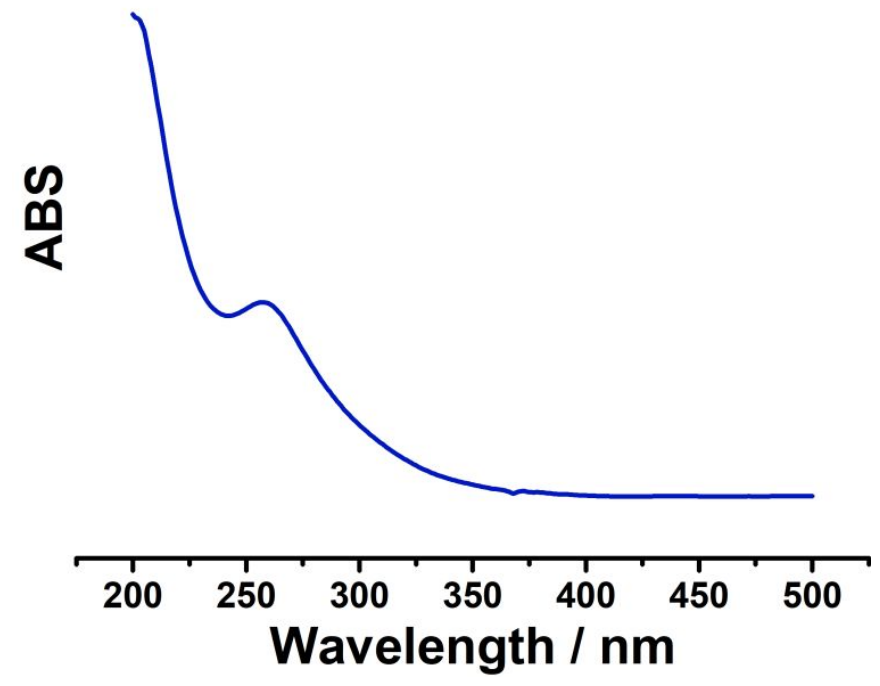

Figure S2. Time-dependent UV-vis absorption spectra of $\mathbf{F e}_{\mathbf{1 5}} \mathbf{P}_{\mathbf{4}}\left(\mathbf{S i W}_{\mathbf{9}}\right)_{\mathbf{4}}$. 


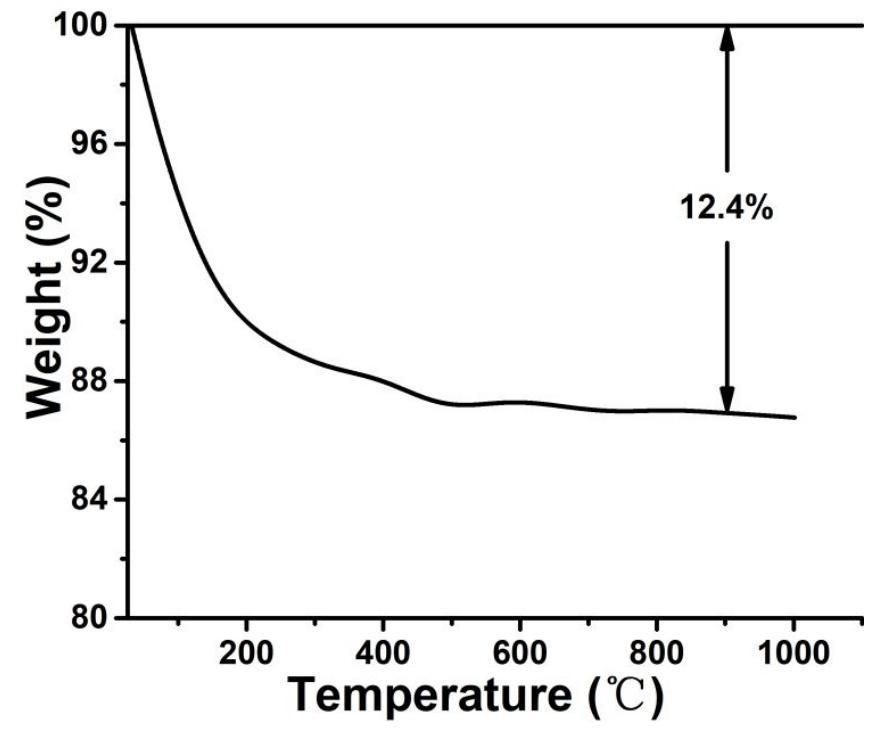

Figure S3. Thermogravimetric analysis of $\mathrm{Na}_{21}-\mathbf{F e}_{15} \mathbf{P}_{\mathbf{4}}\left(\mathbf{S i W}_{\mathbf{9}}\right)_{\mathbf{4}}$. The calculated weight loss percentage $(12.4 \%)$ corresponds to 85 hydration water molecules per formula unit.

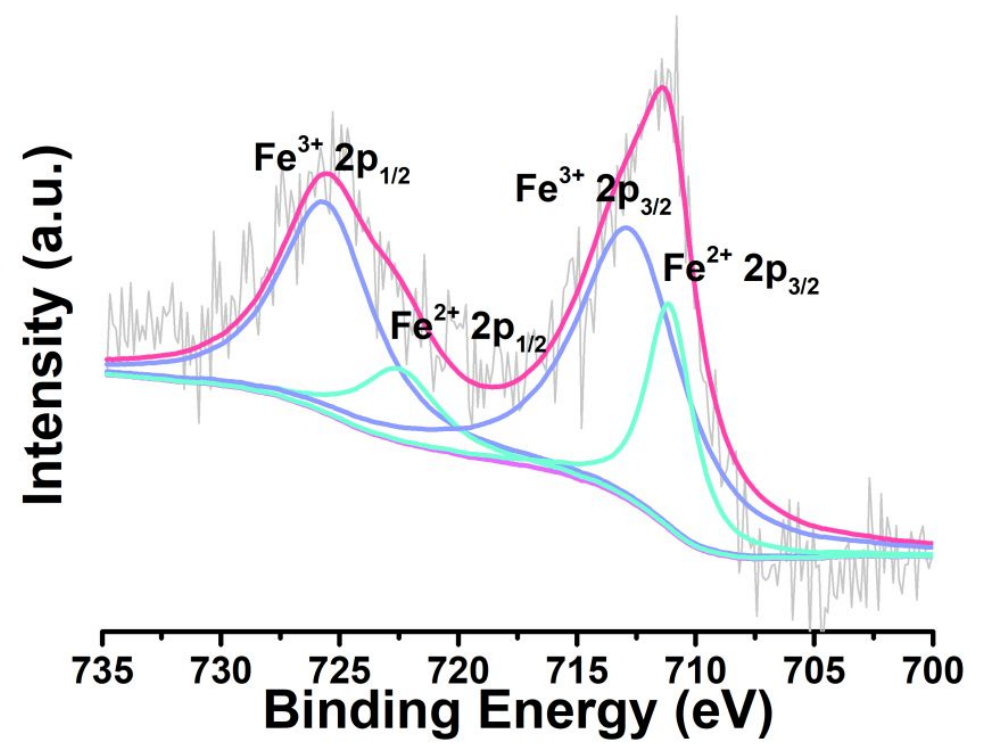

Figure S4. X-ray photoelectron spectroscopy of $\mathrm{Fe}_{15} \mathbf{P}_{\mathbf{4}}\left(\mathrm{SiW}_{\mathbf{9}}\right)_{4}$. 

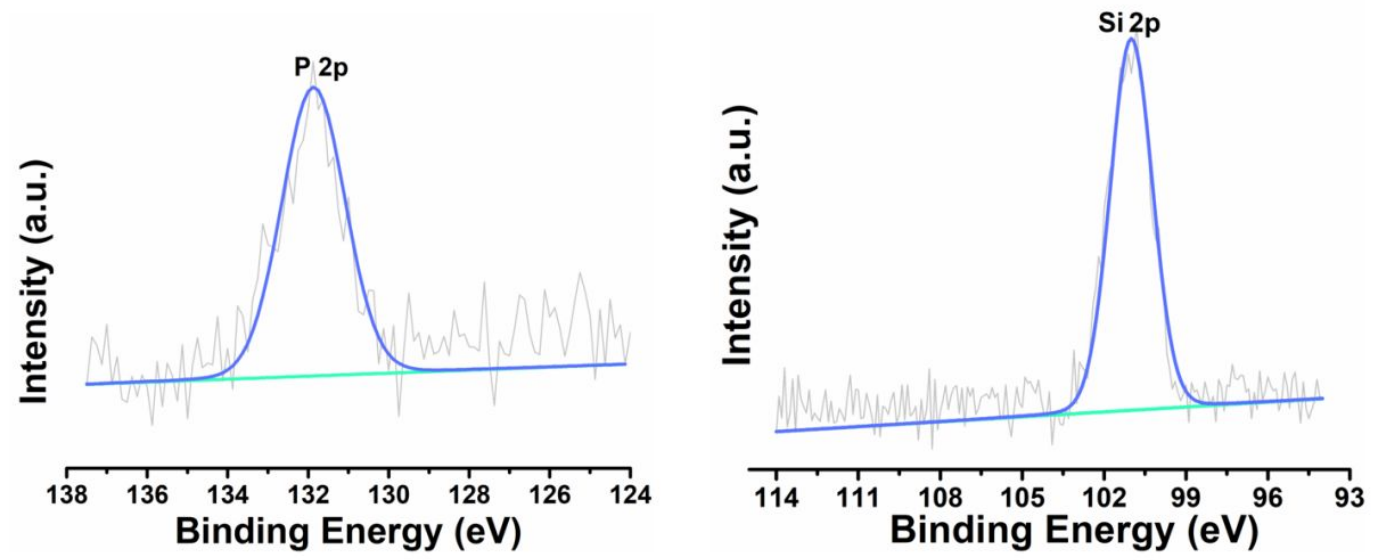

Figure S5. X-ray photoelectron spectroscopy of $\mathbf{S i}$ and $\mathbf{P}$ in the $\mathbf{F e}_{\mathbf{1 5}} \mathbf{P}_{\mathbf{4}}\left(\mathrm{SiW}_{\mathbf{9}}\right)_{\mathbf{4}}$.
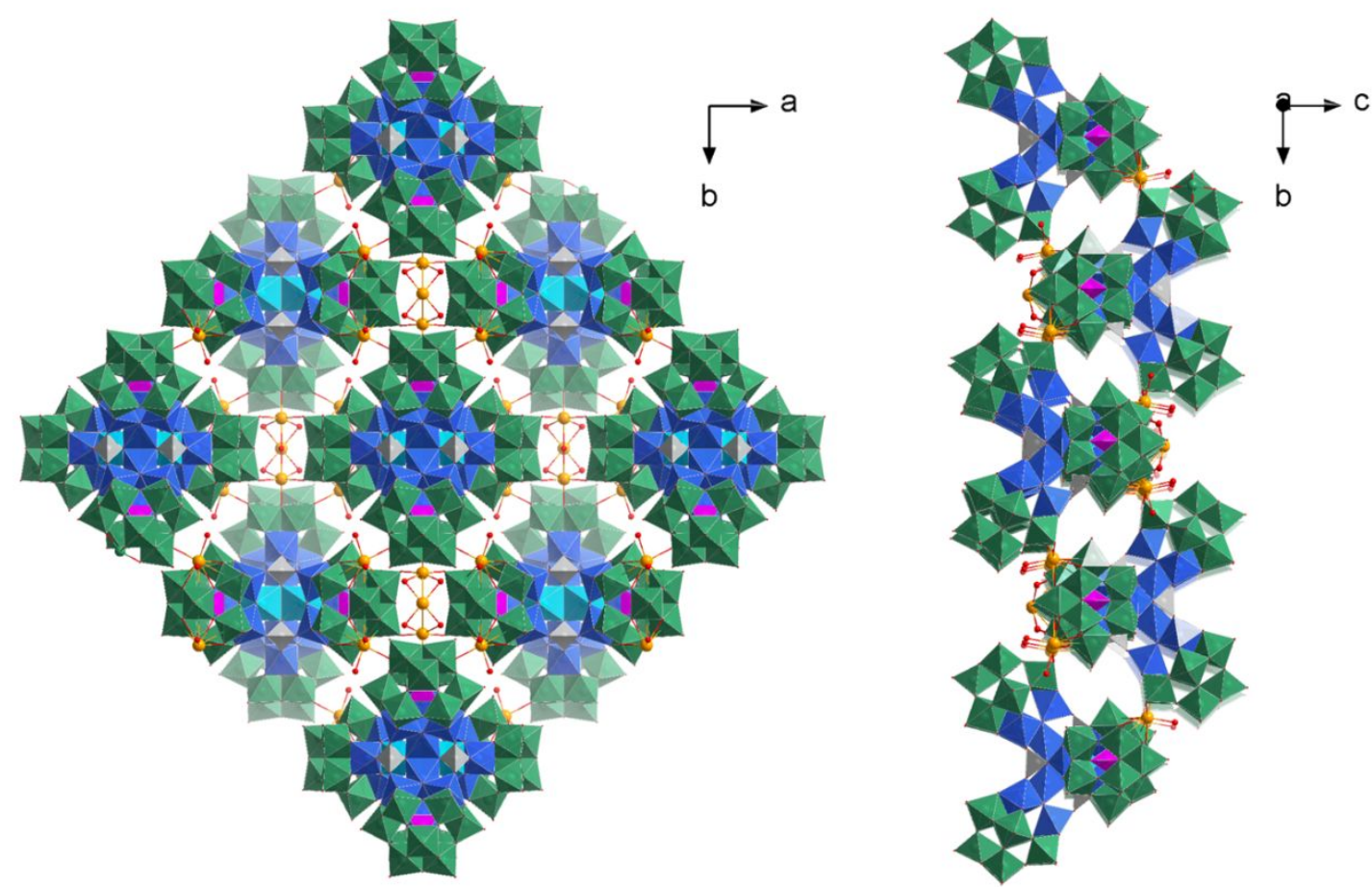

Figure S6. Three-dimensional packing structure of the building blocks of polyoxoanion $\mathrm{Fe}_{15} \mathbf{P}_{\mathbf{4}}\left(\mathrm{SiW}_{\mathbf{9}}\right)_{4}$. Color code: $\mathrm{WO}_{6}=$ green octahedra; $\mathrm{PO}_{4}=$ gray tetrahedral; $\mathrm{SiO}_{4}=$ purple tetrahedra; $\mathrm{FeO}_{6}=$ dark blue octahedron; disordered $\mathrm{FeO}_{6}=$ wathet octahedron; $\mathrm{O}=$ red balls. $\mathrm{Na}=$ yellow balls. 

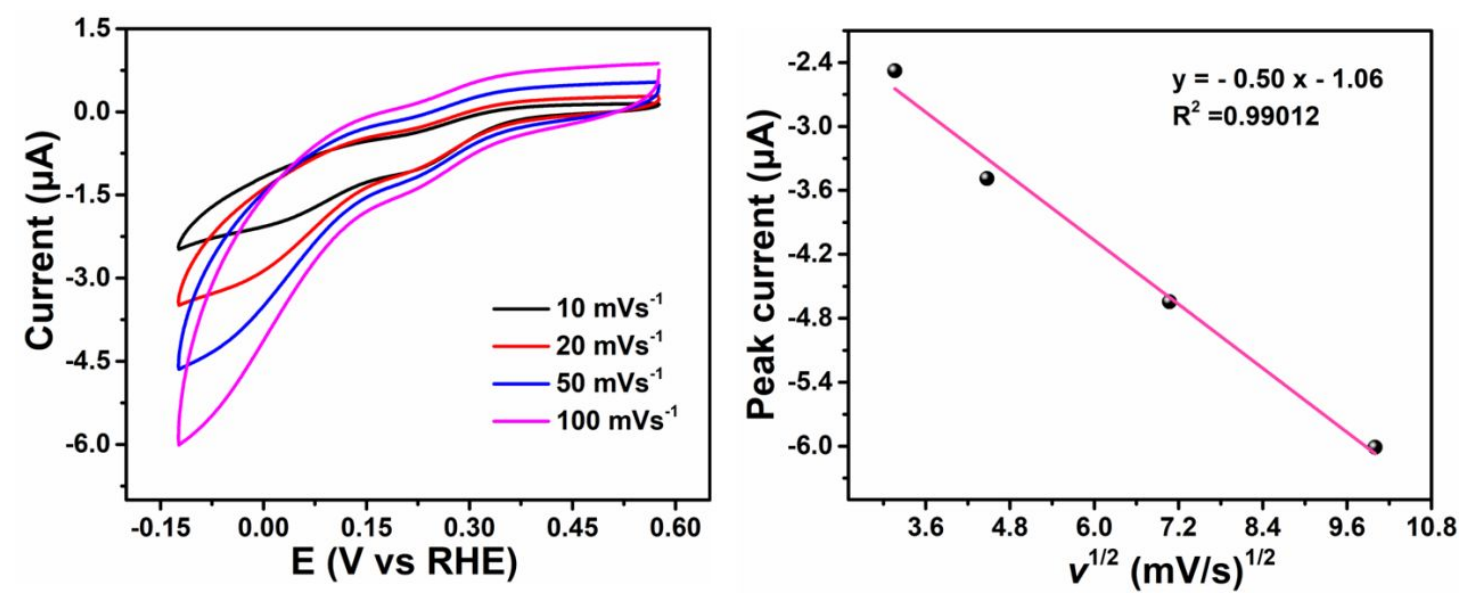

Figure S7. Left: Cyclic voltammograms of $\mathbf{F e}_{1_{5}} \mathbf{P}_{\mathbf{4}}\left(\mathrm{SiW}_{\mathbf{9}}\right)_{4}$ at various scan rates, the redox peak gradually becomes obvious when the scan rates increase from 10 to $100 \mathrm{mV} \mathrm{s}^{-1}$, Right: the plot of minimum anodic peak currents versus the square root of scan rates. Conditions: $2 \mathrm{mM}$ polyoxoanion $\mathbf{F e}_{15} \mathbf{P}_{4}\left(\mathrm{SiW}_{9}\right)_{4}, 5 \mathrm{~mL}$ of $0.5 \mathrm{M} \mathrm{Na}_{2} \mathrm{SO}_{4}$ solution ( $\left.\mathrm{pH}=6.22\right)$; glassy carbon working electrode, $\mathrm{Pt}$ wire auxiliary electrode, $\mathrm{Ag} / \mathrm{AgCl}(3 \mathrm{M} \mathrm{KCl})$ reference electrode, $\mathrm{T}=25^{\circ} \mathrm{C}$.

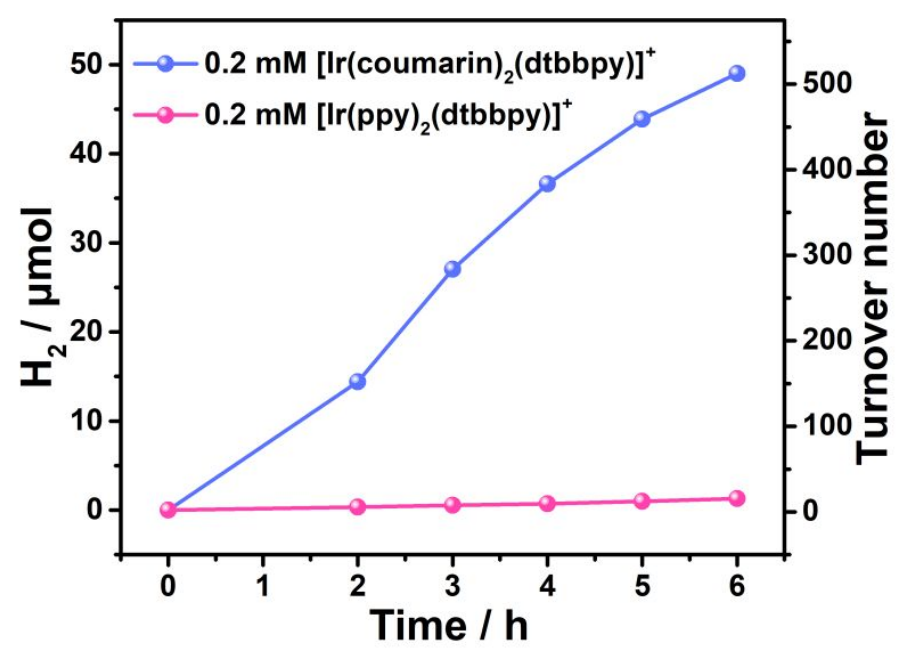

Figure S8. Photocatalytic $\mathrm{H}_{2}$ evolution using different photosensitizer. Conditions: $0.2 \mathrm{mM}$ photosensitizer, 0.25 M TEOA, 1.4 M H $\mathrm{M}_{2} \mathrm{O}, 16 \mu \mathrm{M} \mathrm{Fe}_{\mathbf{1 5}} \mathbf{P}_{\mathbf{4}}\left(\mathbf{S i W}_{\mathbf{9}}\right)_{4}$ catalyst, $\mathrm{CH}_{3} \mathrm{CN} / \mathrm{DMF}(1: 3 \mathrm{v} / \mathrm{v}$, ) deaerated with $\mathrm{Ar} / \mathrm{CH}_{4}$ (4:1 v/v), LED light $455 \mathrm{~nm}$ (red); $300 \mathrm{~W}$ Xe lamp with $400 \mathrm{~nm}$ cut-off filter (blue). 


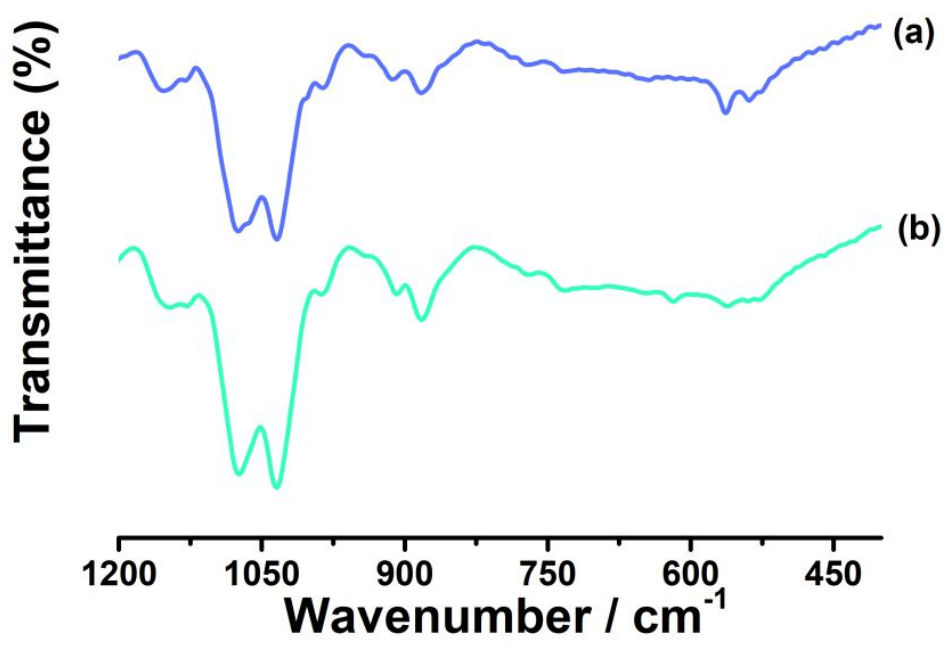

Figure S9. FT-IR spectra of the isolated photosensitizer-Fe ${ }_{15} \mathbf{P}_{\mathbf{4}}\left(\mathbf{S i W}_{\mathbf{9}}\right)_{4}$ adducts obtained by removing the reaction solvent (a) before (blue) and (b) after (green) photocatalysis for 60 hours, $\sim 2 \mathrm{wt} \%$ in $\mathrm{KBr}$.

Table S1. Selected bond lengths $[\AA]$ and angles $\left[{ }^{\circ}\right]$ for $\mathbf{F e}_{\mathbf{1 5}} \mathbf{P}_{\mathbf{4}}\left(\mathbf{S i W}_{\mathbf{9}}\right)_{\mathbf{4}}$.

\begin{tabular}{llll}
\hline $\mathrm{Fe}(1)-\mathrm{O}(24)$ & $1.947(19)$ & $\mathrm{Fe}(4)-\mathrm{O}(31)$ & $1.938(7)$ \\
$\mathrm{Fe}(1)-\mathrm{O}(21)$ & $1.965(13)$ & $\mathrm{Fe}(4)-\mathrm{O}(28)$ & $1.955(13)$ \\
$\mathrm{Fe}(1)-\mathrm{O}(21) \# 1$ & $1.965(13)$ & $\mathrm{Fe}(4)-\mathrm{O}(30)$ & $1.966(13)$ \\
$\mathrm{Fe}(1)-\mathrm{O}(20) \# 1$ & $2.023(12)$ & $\mathrm{Fe}(4)-\mathrm{O}(33)$ & $2.013(12)$ \\
$\mathrm{Fe}(1)-\mathrm{O}(20)$ & $2.023(12)$ & $\mathrm{Fe}(4)-\mathrm{O}(32)$ & $2.050(11)$ \\
$\mathrm{Fe}(1)-\mathrm{O}(17)$ & $2.169(17)$ & $\mathrm{Fe}(4)-\mathrm{O}(41)$ & $2.210(11)$ \\
$\mathrm{Fe}(2)-\mathrm{O}(22)$ & $2.191(19)$ & $\mathrm{Fe}(5)-\mathrm{O}(26) \# 2$ & $2.270(16)$ \\
$\mathrm{Fe}(2)-\mathrm{O}(26)$ & $2.22(2)$ & $\mathrm{Fe}(5)-\mathrm{O}(26)$ & $2.270(16)$ \\
$\mathrm{Fe}(2)-\mathrm{O}(21)$ & $2.217(13)$ & $\mathrm{Fe}(5)-\mathrm{O}(30) \# 3$ & $2.298(13)$ \\
$\mathrm{Fe}(2)-\mathrm{O}(21) \# 1$ & $2.217(13)$ & $\mathrm{Fe}(5)-\mathrm{O}(30)$ & $2.298(13)$ \\
$\mathrm{Fe}(2)-\mathrm{O}(25) \# 2$ & $2.238(17)$ & $\mathrm{Fe}(5)-\mathrm{O}(25)$ & $2.31(3)$ \\
$\mathrm{Fe}(2)-\mathrm{O}(25)$ & $2.238(17)$ & $\mathrm{Fe}(5)-\mathrm{O}(31)$ & $2.328(19)$ \\
$\mathrm{Fe}(3)-\mathrm{O}(23)$ & $1.949(14)$ & $\mathrm{Fe}(6)-\mathrm{O}(30) \# 3$ & $1.932(12)$ \\
$\mathrm{Fe}(3)-\mathrm{O}(21)$ & $1.951(13)$ & $\mathrm{Fe}(6)-\mathrm{O}(30)$ & $1.932(12)$ \\
$\mathrm{Fe}(3)-\mathrm{O}(22)$ & $1.966(7)$ & $\mathrm{Fe}(6)-\mathrm{O}(27)$ & $1.96(2)$ \\
$\mathrm{Fe}(3)-\mathrm{O}(19)$ & $2.020(12)$ & $\mathrm{Fe}(6)-\mathrm{O}(29) \# 3$ & $2.026(12)$ \\
$\mathrm{Fe}(3)-\mathrm{O}(18)$ & $2.048(13)$ & $\mathrm{Fe}(6)-\mathrm{O}(29)$ & $2.026(12)$ \\
$\mathrm{Fe}(3)-\mathrm{O}(16)$ & $2.192(11)$ & $\mathrm{Fe}(6)-\mathrm{O}(42)$ & $2.209(16)$ \\
$\mathrm{O}(24)-\mathrm{Fe}(1)-\mathrm{O}(21)$ & $98.2(5)$ & $\mathrm{O}(31)-\mathrm{Fe}(4)-\mathrm{O}(28)$ & $98.7(6)$ \\
$\mathrm{O}(24)-\mathrm{Fe}(1)-\mathrm{O}(21) \# 1$ & $98.2(5)$ & $\mathrm{O}(31)-\mathrm{Fe}(4)-\mathrm{O}(30)$ & $89.0(6)$ \\
$\mathrm{O}(21)-\mathrm{Fe}(1)-\mathrm{O}(21) \# 1$ & $86.8(7)$ & $\mathrm{O}(28)-\mathrm{Fe}(4)-\mathrm{O}(30)$ & $97.5(5)$ \\
$\mathrm{O}(24)-\mathrm{Fe}(1)-\mathrm{O}(20) \# 1$ & $87.8(5)$ & $\mathrm{O}(31)-\mathrm{Fe}(4)-\mathrm{O}(33)$ & $91.6(6)$ \\
$\mathrm{O}(21)-\mathrm{Fe}(1)-\mathrm{O}(20) \# 1$ & $174.0(6)$ & $\mathrm{O}(28)-\mathrm{Fe}(4)-\mathrm{O}(33)$ & $91.0(5)$ \\
\hline & & &
\end{tabular}




\begin{tabular}{|c|c|c|c|}
\hline $\mathrm{O}(21) \# 1-\mathrm{Fe}(1)-\mathrm{O}(20) \# 1$ & $92.4(5)$ & $\mathrm{O}(30)-\mathrm{Fe}(4)-\mathrm{O}(33)$ & $171.3(5)$ \\
\hline $\mathrm{O}(24)-\mathrm{Fe}(1)-\mathrm{O}(20)$ & $87.8(5)$ & $\mathrm{O}(31)-\mathrm{Fe}(4)-\mathrm{O}(32)$ & $171.9(6)$ \\
\hline $\mathrm{O}(21)-\mathrm{Fe}(1)-\mathrm{O}(20)$ & $92.4(5)$ & $\mathrm{O}(28)-\mathrm{Fe}(4)-\mathrm{O}(32)$ & $89.4(5)$ \\
\hline $\mathrm{O}(21) \# 1-\mathrm{Fe}(1)-\mathrm{O}(20)$ & $174.0(6)$ & $\mathrm{O}(30)-\mathrm{Fe}(4)-\mathrm{O}(32)$ & $90.7(5)$ \\
\hline $\mathrm{O}(20) \# 1-\mathrm{Fe}(1)-\mathrm{O}(20)$ & $87.8(7)$ & $\mathrm{O}(33)-\mathrm{Fe}(4)-\mathrm{O}(32)$ & $87.5(5)$ \\
\hline $\mathrm{O}(24)-\mathrm{Fe}(1)-\mathrm{O}(17)$ & $158.7(7)$ & $\mathrm{O}(31)-\mathrm{Fe}(4)-\mathrm{O}(41)$ & $95.8(5)$ \\
\hline $\mathrm{O}(21)-\mathrm{Fe}(1)-\mathrm{O}(17)$ & $97.2(5)$ & $\mathrm{O}(28)-\mathrm{Fe}(4)-\mathrm{O}(41)$ & $161.2(5)$ \\
\hline $\mathrm{O}(21) \# 1-\mathrm{Fe}(1)-\mathrm{O}(17)$ & $97.2(5)$ & $\mathrm{O}(30)-\mathrm{Fe}(4)-\mathrm{O}(41)$ & $94.7(5)$ \\
\hline $\mathrm{O}(20) \# 1-\mathrm{Fe}(1)-\mathrm{O}(17)$ & $77.0(5)$ & $\mathrm{O}(33)-\mathrm{Fe}(4)-\mathrm{O}(41)$ & $76.6(4)$ \\
\hline $\mathrm{O}(20)-\mathrm{Fe}(1)-\mathrm{O}(17)$ & $77.0(5)$ & $\mathrm{O}(32)-\mathrm{Fe}(4)-\mathrm{O}(41)$ & $76.1(4)$ \\
\hline $\mathrm{O}(22)-\mathrm{Fe}(2)-\mathrm{O}(26)$ & $174.1(8)$ & $\mathrm{O}(26) \# 2-\mathrm{Fe}(5)-\mathrm{O}(26)$ & $84.0(9)$ \\
\hline $\mathrm{O}(22)-\mathrm{Fe}(2)-\mathrm{O}(21)$ & $74.7(5)$ & $\mathrm{O}(26) \# 2-\mathrm{Fe}(5)-\mathrm{O}(30) \# 3$ & $102.1(5)$ \\
\hline $\mathrm{O}(26)-\mathrm{Fe}(2)-\mathrm{O}(21)$ & $100.7(6)$ & $\mathrm{O}(26)-\mathrm{Fe}(5)-\mathrm{O}(30) \# 3$ & $173.2(6)$ \\
\hline $\mathrm{O}(22)-\mathrm{Fe}(2)-\mathrm{O}(21) \# 1$ & $74.7(5)$ & $\mathrm{O}(26) \# 2-\mathrm{Fe}(5)-\mathrm{O}(30)$ & $173.2(6)$ \\
\hline $\mathrm{O}(26)-\mathrm{Fe}(2)-\mathrm{O}(21) \# 1$ & $100.7(6)$ & $\mathrm{O}(26)-\mathrm{Fe}(5)-\mathrm{O}(30)$ & $102.1(5)$ \\
\hline $\mathrm{O}(21)-\mathrm{Fe}(2)-\mathrm{O}(21) \# 1$ & $75.0(6)$ & $\mathrm{O}(30) \# 3-\mathrm{Fe}(5)-\mathrm{O}(30)$ & $71.7(6)$ \\
\hline $\mathrm{O}(22)-\mathrm{Fe}(2)-\mathrm{O}(25) \# 2$ & $101.0(8)$ & $\mathrm{O}(26) \# 2-\mathrm{Fe}(5)-\mathrm{O}(25)$ & $80.8(8)$ \\
\hline $\mathrm{O}(26)-\mathrm{Fe}(2)-\mathrm{O}(25) \# 2$ & $83.6(8)$ & $\mathrm{O}(26)-\mathrm{Fe}(5)-\mathrm{O}(25)$ & $80.8(8)$ \\
\hline $\mathrm{O}(21)-\mathrm{Fe}(2)-\mathrm{O}(25) \# 2$ & $175.4(8)$ & $\mathrm{O}(30) \# 3-\mathrm{Fe}(5)-\mathrm{O}(25)$ & $103.1(6)$ \\
\hline $\mathrm{O}(21) \# 1-\mathrm{Fe}(2)-\mathrm{O}(25) \# 2$ & $102.5(6)$ & $\mathrm{O}(30)-\mathrm{Fe}(5)-\mathrm{O}(25)$ & $103.0(6)$ \\
\hline $\mathrm{O}(22)-\mathrm{Fe}(2)-\mathrm{O}(25)$ & $101.0(8)$ & $\mathrm{O}(26) \# 2-\mathrm{Fe}(5)-\mathrm{O}(31)$ & $103.3(7)$ \\
\hline $\mathrm{O}(26)-\mathrm{Fe}(2)-\mathrm{O}(25)$ & $83.6(8)$ & $\mathrm{O}(26)-\mathrm{Fe}(5)-\mathrm{O}(31)$ & $103.3(7)$ \\
\hline $\mathrm{O}(21)-\mathrm{Fe}(2)-\mathrm{O}(25)$ & $102.5(6)$ & $\mathrm{O}(30) \# 3-\mathrm{Fe}(5)-\mathrm{O}(31)$ & $72.6(5)$ \\
\hline $\mathrm{O}(21) \# 1-\mathrm{Fe}(2)-\mathrm{O}(25)$ & $175.4(8)$ & $\mathrm{O}(30)-\mathrm{Fe}(5)-\mathrm{O}(31)$ & $72.6(5)$ \\
\hline $\mathrm{O}(25) \# 2-\mathrm{Fe}(2)-\mathrm{O}(25)$ & $79.6(10)$ & $\mathrm{O}(25)-\mathrm{Fe}(5)-\mathrm{O}(31)$ & $174.5(8)$ \\
\hline $\mathrm{O}(23)-\mathrm{Fe}(3)-\mathrm{O}(21)$ & $99.0(6)$ & $\mathrm{O}(30) \# 3-\mathrm{Fe}(6)-\mathrm{O}(30)$ & $88.3(7)$ \\
\hline $\mathrm{O}(23)-\mathrm{Fe}(3)-\mathrm{O}(22)$ & $97.6(6)$ & $\mathrm{O}(30) \# 3-\mathrm{Fe}(6)-\mathrm{O}(27)$ & $98.8(6)$ \\
\hline $\mathrm{O}(21)-\mathrm{Fe}(3)-\mathrm{O}(22)$ & $86.1(6)$ & $\mathrm{O}(30)-\mathrm{Fe}(6)-\mathrm{O}(27)$ & $98.8(6)$ \\
\hline $\mathrm{O}(23)-\mathrm{Fe}(3)-\mathrm{O}(19)$ & $90.3(5)$ & $\mathrm{O}(30) \# 3-\mathrm{Fe}(6)-\mathrm{O}(29) \# 3$ & $91.6(5)$ \\
\hline $\mathrm{O}(21)-\mathrm{Fe}(3)-\mathrm{O}(19)$ & $91.9(5)$ & $\mathrm{O}(30)-\mathrm{Fe}(6)-\mathrm{O}(29) \# 3$ & $171.6(5)$ \\
\hline $\mathrm{O}(22)-\mathrm{Fe}(3)-\mathrm{O}(19)$ & $172.0(6)$ & $\mathrm{O}(27)-\mathrm{Fe}(6)-\mathrm{O}(29) \# 3$ & $89.5(6)$ \\
\hline $\mathrm{O}(23)-\mathrm{Fe}(3)-\mathrm{O}(18)$ & $88.2(6)$ & $\mathrm{O}(30) \# 3-\mathrm{Fe}(6)-\mathrm{O}(29)$ & $171.6(5)$ \\
\hline $\mathrm{O}(21)-\mathrm{Fe}(3)-\mathrm{O}(18)$ & $172.8(5)$ & $\mathrm{O}(30)-\mathrm{Fe}(6)-\mathrm{O}(29)$ & $91.5(5)$ \\
\hline $\mathrm{O}(22)-\mathrm{Fe}(3)-\mathrm{O}(18)$ & $92.6(6)$ & $\mathrm{O}(27)-\mathrm{Fe}(6)-\mathrm{O}(29)$ & $89.5(5)$ \\
\hline $\mathrm{O}(19)-\mathrm{Fe}(3)-\mathrm{O}(18)$ & $88.4(5)$ & $\mathrm{O}(29) \# 3-\mathrm{Fe}(6)-\mathrm{O}(29)$ & $87.3(7)$ \\
\hline $\mathrm{O}(23)-\mathrm{Fe}(3)-\mathrm{O}(16)$ & $160.5(5)$ & $\mathrm{O}(30) \# 3-\mathrm{Fe}(6)-\mathrm{O}(42)$ & $94.9(5)$ \\
\hline $\mathrm{O}(21)-\mathrm{Fe}(3)-\mathrm{O}(16)$ & $95.8(5)$ & $\mathrm{O}(30)-\mathrm{Fe}(6)-\mathrm{O}(42)$ & $94.9(5)$ \\
\hline $\mathrm{O}(22)-\mathrm{Fe}(3)-\mathrm{O}(16)$ & $95.9(6)$ & $\mathrm{O}(27)-\mathrm{Fe}(6)-\mathrm{O}(42)$ & $160.9(7)$ \\
\hline $\mathrm{O}(19)-\mathrm{Fe}(3)-\mathrm{O}(16)$ & $76.5(5)$ & $\mathrm{O}(29) \# 3-\mathrm{Fe}(6)-\mathrm{O}(42)$ & $76.7(5)$ \\
\hline $\mathrm{O}(18)-\mathrm{Fe}(3)-\mathrm{O}(16)$ & $77.3(5)$ & $\mathrm{O}(29)-\mathrm{Fe}(6)-\mathrm{O}(42)$ & $76.7(5)$ \\
\hline
\end{tabular}


Table S2. Bond-valence sum (BVS) of $\mathrm{Fe}_{15} \mathrm{P}_{4}\left(\mathrm{SiW}_{9}\right)_{4}$.

\begin{tabular}{lllllll}
\hline Element & $\mathbf{P}_{\mathbf{1}}$ & $\mathbf{P}_{\mathbf{2}}$ & $\mathbf{S i}_{\mathbf{1}}$ & $\mathbf{S i}_{\mathbf{2}}$ & & \\
BVS value & 4.99 & 4.97 & 3.95 & 4.01 & & \\
\hline Element & $\mathbf{F e}_{\mathbf{1}}$ & $\mathbf{F e}_{\mathbf{2}}$ & $\mathbf{F e}_{\mathbf{3}}$ & $\mathbf{F e}_{\mathbf{4}}$ & $\mathbf{F e}_{\mathbf{5}}$ & $\mathbf{F e}_{\mathbf{6}}$ \\
BVS value & 3.04 & 1.73 & 3.03 & 3.02 & 1.41 & 3.09 \\
\hline $\boldsymbol{\mu}_{\mathbf{3}} \mathbf{- O}$ & $\mathbf{O}_{\mathbf{2 1}}$ & $\mathbf{O}_{\mathbf{2 2}}$ & $\mathbf{O}_{\mathbf{3 0}}$ & $\mathbf{O}_{\mathbf{3 1}}$ & & \\
BVS value & 1.43 & 1.43 & 1.41 & 1.42 & & \\
\hline
\end{tabular}

Table S3. Comparison of recent literature results for various Fe-POMs catalysts concerning the photocatalytic HER.

\begin{tabular}{|c|c|c|c|c|c|c|}
\hline Catalyst & Co-catalyst & Light source & Photosensitizer & $\begin{array}{l}\text { Sacrificial } \\
\text { reagent }\end{array}$ & TON & Ref. \\
\hline$\left\{\mathrm{FeW}_{12} \mathrm{O}_{40}\right\}$ & $\begin{array}{c}\mathrm{Pt} \\
\text { None }\end{array}$ & $\begin{array}{l}\text { High pressure Hg or } \\
150 \mathrm{~W} \text { Xe Lamp }\end{array}$ & None & $\mathrm{CH}_{3} \mathrm{OH}$ & $\begin{array}{l}1.90 \\
1.56\end{array}$ & 1 \\
\hline$\left\{\mathrm{TiW}_{11} \mathrm{Fe}\right\}$ & None & $\begin{array}{l}\text { Simulated solar light } \\
\qquad(350-760 \mathrm{~nm})\end{array}$ & None & $\begin{array}{l}\text { Polyvinyl } \\
\text { alcohol }\end{array}$ & 1.88 & 2 \\
\hline$\left\{\mathrm{FeTa}_{6} \mathrm{Si}_{2} \mathbf{W}_{18}\right\}$ & None & 300 W Hg lamp & None & $\mathrm{CH}_{3} \mathrm{OH}$ & 60 & 3 \\
\hline$\left\{\mathrm{Fe}_{6} \mathrm{Se}_{6} \mathrm{~W}_{34}\right\}$ & None & $500 \mathrm{~W}$ Hg lamp & None & $\mathrm{CH}_{3} \mathrm{OH}$ & 181 & 4 \\
\hline$\left\{\mathrm{Fe}_{10} \mathrm{Se}_{8} \mathrm{~W}_{62}\right\}$ & None & 500W Hg lamp & None & $\mathrm{CH}_{3} \mathrm{OH}$ & 193 & 4 \\
\hline$\left\{\mathrm{Fe}_{15} \mathbf{P}_{\mathbf{4}}\left(\mathrm{SiW}_{9}\right)_{4}\right\}$ & None & $\begin{array}{l}300 \mathrm{~W} \text { Xe lamp } \\
\text { (400 nm cut-off } \\
\text { filter) }\end{array}$ & $\begin{array}{c}{\left[\operatorname{Ir}(\text { coumarin })_{2}(\right.} \\
\text { dtbbpy })]\left[\mathrm{PF}_{6}\right]\end{array}$ & TEOA & 986 & $\begin{array}{l}\text { This } \\
\text { work }\end{array}$ \\
\hline
\end{tabular}

\section{References}

(1) Ioannidis, A.; Papaconstantinou, E. Photocatalytic generation of hydrogen by 1:12 heteropolytungstates with concomitant oxidation of organic compounds. Inorg. Chem. 1985, 24, 439441.

(2) Shang, X.; Liu, R.; Zhang, G.; Zhang, S.; Cao, H.; Gu, Z. Artificial photosynthesis for solar hydrogen generation over transition-metal substituted Keggin-type titanium tungstate. New J. Chem. 2014, 38, 1315-1320.

(3) Huang, P.; Han, X.-G.; Li, X.-L.; Qin, C.; Wang, X.-L.; Su, Z.-M. Self-assembly and photocatalytic properties of $\mathrm{Ta} / \mathrm{W}$ mixed-addendum polyoxometalate and transition-metal cations. CrystEngComm. 2016, 18, 8722-8725.

(4) Chen, W. C.; Qin, C.; Wang, X. L.; Li, Y. G.; Zang, H. Y.; Jiao, Y. Q.; Huang, P.; Shao, K. Z.; Su, Z. M.; Wang, E. B. Assembly of Fe-substituted Dawson-type nanoscale selenotungstate clusters with photocatalytic $\mathrm{H}_{2}$ evolution activity. Chem. Commun. 2014, 50, 13265-13267. 